\title{
Open
}

\section{Diagnostic exome sequencing provides a molecular diagnosis for a significant proportion of patients with epilepsy}

\author{
Katherine L. Helbig, MS', Kelly D. Farwell Hagman, MS', Deepali N. Shinde, PhD'1, \\ Cameron Mroske, MSc'1, Zöe Powis, MS', Shuwei Li, $\mathrm{PhD}^{2}$, Sha Tang, $\mathrm{PhD}^{1}$ and Ingo Helbig, MD ${ }^{3,4}$
}

Purpose: To assess the yield of diagnostic exome sequencing (DES) and to characterize the molecular findings in characterized and novel disease genes in patients with epilepsy.

Methods: In an unselected sample of 1,131 patients referred for DES, overall results were compared between patients with and without epilepsy. DES results were examined based on age of onset and epilepsy diagnosis.

Results: Positive/likely positive results were identified in 112/293 (38.2\%) epilepsy patients compared with $210 / 732$ (28.7\%) patients without epilepsy $(P=0.004)$. The diagnostic yield in characterized disease genes among patients with epilepsy was 33.4\% (105/314). KCNQ2, MECP2, FOXG1, IQSEC2, KMT2A, and STXBP1 were most commonly affected by de novo alterations. Patients with epileptic encephalopathies had the highest rate of positive findings (43.4\%).
A likely positive novel genetic etiology was proposed in 14/200 (7\%) patients with epilepsy; this frequency was highest in patients with epileptic encephalopathies (17\%). Three genes (COQ4, DNM1, and $P U R A$ ) were initially reported as likely positive novel disease genes and were subsequently corroborated in independent peer-reviewed publications.

Conclusion: DES with analysis and interpretation of both characterized and novel genetic etiologies is a useful diagnostic tool in epilepsy, particularly in severe early-onset epilepsy. The reporting on novel genetic etiologies may further increase the diagnostic yield.

Genet Med advance online publication 21 January 2016

Key Words: diagnostic yield; epilepsy; epileptic encephalopathy; seizure; whole-exome sequencing

\section{INTRODUCTION}

Since its introduction in 2011, diagnostic exome sequencing (DES) has proven instrumental in providing a molecular diagnosis for many patients with a broad spectrum of previously undiagnosed genetic disorders because it can comprehensively sequence the coding regions of the $\sim 20,000$ genes in the genome. ${ }^{1}$ DES offers a cost-effective and comprehensive method to detect underlying alterations in presumed genetic disorders and may significantly shorten the so-called diagnostic odyssey that many patients with rare disorders experience, resulting in a faster time to diagnosis and overall reduction in health-care costs. ${ }^{2,3}$ In a 2012 policy statement, the American College of Medical Genetics and Genomics endorsed the use of genomic sequencing technologies in clinical practice for patients with suspected genetic disorders when (i) the patient presents with a phenotype that is inconsistent with currently testable syndromes, (ii) the patient presents with a phenotype that is consistent with a disorder with many genetic causes, or (iii) no cause has been discovered despite extensive testing. ${ }^{4}$

Although the overall diagnostic yield from smaller selected patient cohorts varies widely in neurodevelopmental disorders, data from large unselected laboratory cohorts estimate that the overall diagnostic yield of DES ranges from 25 to $37 \%$ (refs. 5-7). This is in line with a reported diagnostic yield of $27-45 \%$ for patients with a broad spectrum of neurodevelopmental disorders. ${ }^{3,8,9}$ However, initial studies also demonstrate that the diagnostic yield of DES may depend on the particular patient phenotype. ${ }^{10-12}$

Seizures are a common comorbidity of many known Mendelian disorders, and many epilepsy syndromes have a strong genetic component. ${ }^{13,14}$ Recent data show that de novo mutations play an important role in early-onset epileptic encephalopathies. ${ }^{15,16}$ Furthermore, epilepsy is a common feature of neurodevelopmental disorders, including autism spectrum disorder and intellectual disability, with many shared causative genes such as CHD2, GRIN2B, SCN2A, and SYNGAP1. ${ }^{15,17-22}$

Several recent studies have highlighted the usefulness of DES in clinical pediatric neurology practice, emphasizing a high diagnostic rate and impact on genetic counseling and patient management. ${ }^{8,9,23}$ Data from small cohorts of patients with severe epilepsies also highlight the utility of DES for improving molecular diagnoses. ${ }^{24-26}$ However, these studies rely on data from small, highly selected patient cohorts and may not reflect the diagnostic yield in an unselected sample of patients with epilepsy.

${ }^{1}$ Division of Clinical Genomics, Ambry Genetics, Aliso Viejo, California, USA; ${ }^{2}$ Department of Bioinformatics, Ambry Genetics, Aliso Viejo, California, USA; ${ }^{3}$ Division of Neurology, The Children's Hospital of Philadelphia, Philadelphia, Pennsylvania, USA; ${ }^{4}$ Department of Neuropediatrics, University Medical Center Schleswig-Holstein, Christian-Albrechts-University, Kiel, Germany. Correspondence: Katherine L. Helbig (khelbig@ambrygen.com) 
To better elucidate the diagnostic yield of DES for patients with epilepsy, we evaluated DES results in 314 consecutive patients with epilepsy referred to our laboratory.

\section{Patient population}

\section{PATIENTS AND METHODS}

Patients were ascertained sequentially through clinical samples sent to Ambry Genetics Laboratory for DES. Clinicians were also encouraged to refer all first-degree relatives and other informative family members for testing. Solutions Institutional Review Board determined the study to be exempt from the Office for Human Research Protections Regulations for the Protection of Human Subjects (45 CFR 46) under category 4. Retrospective data analysis of anonymized data exempted the study from the requirement of receiving consent from patients.

Patients' clinical and testing histories, along with pedigrees provided by referring physicians, were reviewed and summarized for each case by a team of genetic counselors. When possible, information regarding the patients' clinical seizure semiology, EEG findings, and brain imaging reports were reviewed by a child neurologist and a genetic counselor with experience in clinical epilepsy genetics. International League Against Epilepsy criteria were used for epilepsy syndrome classification. ${ }^{27}$

\section{Whole-exome sequencing}

Genomic DNA was isolated from whole blood from all probands and accompanying family members. Exome library preparation, sequencing, bioinformatics, and data analysis were performed as previously described. ${ }^{7}$ Briefly, samples were prepared using either the SureSelect Target Enrichment System (Agilent Technologies, Santa Clara, CA) ${ }^{28}$ or Roche NimbleGen VCRome Exome System (Madison, WI) and sequenced using paired-end, 100-cycle chemistry on the Illumina HiSeq 2000 or 2500 (Illumina, San Diego, CA). Sequence data were aligned to the reference human genome (GRCh37), and variant calls were generated using CASAVA and Pindel. ${ }^{29}$ Stepwise filtering included the removal of common single-nucleotide polymorphisms, intergenic and $3^{\prime} / 5^{\prime}$ UTR variants, nonsplice-related intronic variants, and synonymous variants. Variants were filtered further based on family history and possible inheritance models. Data were annotated with the Ambry Variant Analyzer tool. ${ }^{30}$ All samples were required to meet minimum quality standards, with at least $90 \%$ of bases covered at $\geq 10 \times$ and base call quality scores $\geq Q 20$, which translates to a base-calling error rate of 1:100. Identified candidate alterations were confirmed using automated fluorescence dideoxy sequencing.

\section{Data analysis}

Characterized disease genes were defined as genes known to underlie at least one Mendelian genetic condition, whereas novel disease genes were defined as genes not currently known to underlie a Mendelian genetic condition. A novel genetic etiology describes the discovery of a potentially new gene-disease relationship and/or mechanism not previously proposed or with limited evidence based on ClinGen clinical validity assessment criteria (http://www.clinicalgenome.org/knowledge-curation/ gene-curation/clinical-validity-classifications/).

As such, the overall primary DES results categories were classified as positive/likely positive characterized gene findings, uncertain characterized gene findings, likely positive or possibly positive novel genetic etiologies, or negative. Secondary or incidental findings were not included in this analysis. Overall DES results were considered positive/likely positive if a pathogenic/likely pathogenic mutation was identified with positive phenotypic overlap with the patient's primary indication for testing. Analysis of novel genetic etiologies followed an internally developed assessment scheme that evaluates evidence including human microdeletion/duplication syndromes, gene function and expression profiles, colocalization and interaction with genes known to cause similar phenotypes, in vivo animal models, gene family and pathway information, and possible mutational mechanisms inferred from distribution of variants in control populations (Supplementary Materials and Methods online). Classification of candidate gene alterations identified in both characterized genes and novel genetic etiologies followed Ambry's clinical variant classification scheme (Supplementary Materials and Methods), ${ }^{30}$ which incorporates published recommendations and guidelines by the American College of Medical Genetics and Genomics and the Association for Molecular Pathology. ${ }^{31}$ Different DES testing strategies were applied in the diagnostic workflow depending on which test was requested. The strategies mainly differ with respect to whether characterized genes were analyzed first or whether both characterized and novel disease genes were considered initially: "first-tier" or "characterized gene only" analysis involved proband-only exome sequencing and analysis of characterized disease genes only. "Reflexive" or "step-wise" analysis entailed analysis of characterized disease genes and, if the results were negative, analysis of novel genetic etiologies. Finally, the "complete" or "nonreflexive" analysis included parallel analysis of characterized and novel genetic etiologies. Accordingly, not all patients were analyzed for novel disease genes, and different denominators were used for the assessment of overall positive rates. To account for the different analysis approaches used, the overall positive rate excluded probands in whom only characterized disease genes were analyzed (excluding patients with "first-tier" analysis only). Calculation of diagnostic rates in characterized disease genes was based on all probands, regardless of analysis strategy. Calculation of novel genetic etiology detection rates was based on the number of probands in whom analysis of both characterized and novel genetic etiologies was performed. Overall primary DES results categories were compared between patients with epilepsy ("epilepsy group") and patients without epilepsy ("nonepilepsy group") using Fisher's exact test. Overall DES results categories were also examined based on age of seizure onset and epilepsy diagnosis. 
Table 1 Clinical characteristics of 1,131 total probands who underwent diagnostic exome sequencing, including 314 patients with seizures and 817 patients without seizures

\begin{tabular}{|c|c|c|c|c|c|}
\hline \multirow[b]{2}{*}{$\begin{array}{l}\text { Intellectual disability/ } \\
\text { developmental delay }\end{array}$} & \multicolumn{2}{|c|}{$\begin{array}{l}\text { Epilepsy } \\
\text { group }\end{array}$} & \multicolumn{2}{|c|}{$\begin{array}{l}\text { Nonepilepsy } \\
\text { group }\end{array}$} & \multirow{2}{*}{$\begin{array}{c}\begin{array}{c}\boldsymbol{P} \\
\text { value }\end{array} \\
<0.0001\end{array}$} \\
\hline & 282 & $89.8 \%$ & 499 & $61.1 \%$ & \\
\hline Autism & 56 & $17.8 \%$ & 96 & $11.8 \%$ & 0.009 \\
\hline $\begin{array}{l}\text { Abnormal brain MRI } \\
\text { findings }\end{array}$ & 166 & $52.9 \%$ & 232 & $28.4 \%$ & $<0.0001$ \\
\hline $\begin{array}{l}\text { Multiple congenital } \\
\text { anomalies }\end{array}$ & 73 & $23.2 \%$ & 250 & $30.6 \%$ & 0.015 \\
\hline $\begin{array}{l}\text { Positive family history of } \\
\text { seizures }\end{array}$ & 74 & $23.6 \%$ & 65 & $8.0 \%$ & $<0.0001$ \\
\hline \multicolumn{6}{|l|}{ Age at testing } \\
\hline Prenatal & 0 & $0.0 \%$ & 5 & $0.6 \%$ & 0.33 \\
\hline $0-3$ months & 6 & $1.9 \%$ & 29 & $3.5 \%$ & 0.18 \\
\hline $3-12$ months & 21 & $6.7 \%$ & 58 & $7.1 \%$ & 0.90 \\
\hline $1-5$ years & 103 & $32.8 \%$ & 287 & $35.1 \%$ & 0.49 \\
\hline $5-12$ years & 95 & $30.3 \%$ & 193 & $23.6 \%$ & 0.005 \\
\hline $12-18$ years & 51 & $16.2 \%$ & 90 & $11.0 \%$ & 0.021 \\
\hline $18-40$ years & 33 & $10.5 \%$ & 83 & $10.2 \%$ & 0.91 \\
\hline$>40$ years & 5 & $1.6 \%$ & 72 & $8.8 \%$ & 0.0001 \\
\hline \multicolumn{6}{|l|}{ Sex } \\
\hline Male & 167 & $53.2 \%$ & 466 & $57.0 \%$ & 0.256 \\
\hline Female & 147 & $46.8 \%$ & 351 & $43.0 \%$ & - \\
\hline
\end{tabular}

$\mathrm{MRI}$, magnetic resonance imaging.

\section{Logistic regression analysis to account for differences between cohorts}

To assess the predictive value of seizures and to account for clinical differences between the epilepsy and nonepilepsy groups on the overall DES results that may affect the interpretation or our findings, logistic regression analysis was performed using R version 3.1.1. ${ }^{32}$ Overall DES results categories were designated as the response variables and the presence or absence of seizures in the proband was used as the predictor variable. All analyses were adjusted for potential confounding effects of age, sex, intellectual disability, autism, positive brain magnetic resonance imaging findings, multiple congenital anomalies, and family history of seizures in a multiple logistic regression model. For positive/likely positive cases, the inheritance of the identified mutation was determined, when possible, and compared between the epilepsy and nonepilepsy groups as well as across epilepsy diagnosis subgroups.

\section{RESULTS}

\section{Demographics and epilepsy diagnoses}

The epilepsy group comprised a total of $314 / 1,131$ patients (27.8\%) who were determined to have seizures. Demographic characteristics and epilepsy diagnoses are summarized in Tables 1 and 2. The epilepsy and nonepilepsy groups significantly differed with respect to intellectual disability/
Table 2 Epilepsy diagnoses in 314 probands with seizures

\begin{tabular}{lcr} 
Epilepsy diagnoses & $\boldsymbol{n}$ & \multicolumn{1}{c}{$\%$} \\
\hline Epileptic encephalopathies & 89 & $28.3 \%$ \\
Infantile spasms & 37 & $41.6 \%$ \\
Infantile spasms to Lennox-Gastaut & 5 & $5.6 \%$ \\
Lennox-Gastaut syndrome & 4 & $4.5 \%$ \\
Neonatal-onset epileptic encephalopathy & 16 & $18.0 \%$ \\
Infantile-onset epileptic encephalopathy & 15 & $16.9 \%$ \\
Epileptic encephalopathy, ESES-like & 3 & $3.4 \%$ \\
Epileptic encephalopathy, other & 9 & $10.1 \%$ \\
Fever-related seizures & 27 & $8.6 \%$ \\
\hline Focal epilepsies & 41 & $13.1 \%$ \\
Benign Rolandic/Rolandic epilepsy & 2 & $4.9 \%$ \\
Frontal lobe epilepsy & 3 & $7.3 \%$ \\
Temporal lobe epilepsy & 8 & $19.5 \%$ \\
Occipital lobe epilepsy & 2 & $4.9 \%$ \\
Unclassified focal epilepsy & 26 & $63.4 \%$ \\
Generalized epilepsies & 25 & $8.0 \%$ \\
Idiopathic/genetic generalized epilepsy & 9 & $36.0 \%$ \\
Progressive myoclonus epilepsy & 2 & $8.0 \%$ \\
Unclassified generalized epilepsy & 14 & $56.0 \%$ \\
Symptomatic seizures & 3 & $1.0 \%$ \\
Single unprovoked seizure & 5 & $1.6 \%$ \\
Benign familial neonatal seizures & 1 & $0.3 \%$ \\
Unclassifiable epilepsy & 123 & $39.2 \%$ \\
\hline SES, & &
\end{tabular}

ESES, electrical status epilepticus during sleep.

developmental delay, autism, abnormal brain magnetic resonance imaging findings, presence of multiple congenital anomalies, and positive family history of seizures. The epilepsy and nonepilepsy groups did not significantly differ with respect to age or sex. The majority of patients who could be classified had a diagnosis of epileptic encephalopathy $(89 / 314 ; 28.3 \%)$; however, a large proportion of patients were unable to be classified due to lacking or incomplete clinical data (39.2\%). Most patients with epilepsy were referred for exome sequencing between ages 1 and 5 years $(103 / 314 ; 32.8 \%)$.

\section{Prior genetic diagnostic testing}

Because DES is a diagnostic test that is frequently used later in the diagnostic workup, when possible we obtained information on prior genetic testing that had been performed in patients in the epilepsy group. Ninety-four of 314 (29.9\%) patients had negative next-generation sequencing (NGS) panel testing prior to exome sequencing, including negative epilepsy NGS panels in 42/94 patients. The panels used varied widely with respect to the number and type of genes included. The remaining 52/94 patients had other types of NGS panels prior to exome sequencing, including X-linked intellectual disability panels, mitochondrial panels, ataxia panels, and Rett/Angelman panels. Of 314 patients, 250 (79.6\%) had uninformative single-nucleotide polymorphism array or array-comparative genomic hybridization prior to DES. 


\section{Exome sequencing strategy}

For the epilepsy group, DES was performed on the probandparent trios in $254 / 314(80.9 \%)$ cases, on the proband only in $19 / 314(6.1 \%)$ cases, on the proband plus one first-degree relative in $18 / 314(5.7 \%)$ cases, or on the proband and other nonparent first-degree relatives in 23/314 (7.3\%) cases. The exome sequencing strategy was not significantly different for epilepsy cases compared with nonepilepsy cases (Supplementary Table S1 online). Characterized gene-only analysis was performed on $21 / 314(6.7 \%)$ probands in the epilepsy group and on $85 / 817(10.4 \%)$ in the nonepilepsy group. Further analysis strategies for all probands are summarized in Supplementary Table S2 online; patients with epilepsy underwent complete analysis of both characterized genes and novel genetic etiologies more frequently than did patients without epilepsy. Analysis of characterized and novel genetic etiologies was performed on $200 / 314(63.7 \%)$ patients in the epilepsy group and on $521 / 817$ $(63.8 \%)$ patients in the nonepilepsy group.

\section{Positive rate in characterized disease genes}

Positive findings in characterized disease genes were identified in $105 / 314$ (33.4\%) patients with epilepsy, referring to the entire cohort of patients. Mutations in KCNQ2, MECP2, FOXG1, IQSEC2, KMT2A, and STXBP1 were the most frequent findings (Supplementary Table S3 online; see below).

\section{Overall positive rate in both characterized and novel genetic etiologies}

For this analysis, we excluded patients in whom the analysis was a priori limited to characterized genetic etiologies only (i.e., patients in whom "first-tier" analysis was performed; $n=21$; Supplementary Figure $\mathbf{S} 1$ online). In the remaining 293 patients, we identified positive or likely positive findings in 112 patients with epilepsy (38.2\%).

\section{Positive rate in novel genetic etiologies}

In 200/293 (68.3\%) patients, analysis of novel genetic etiologies was performed when no clearly pathogenic mutation in a characterized genetic etiology was identified in the analysis of characterized disease genes (Supplementary Figure S1 online). Fourteen of $200(7.0 \%)$ patients had likely positive findings in novel genetic etiologies (Table 3 ). Possibly positive findings in novel genetic etiologies were found in an additional four patients but were not included in the calculation of overall positive rates.

\section{Comparison of epilepsy with nonepilepsy cases}

Patients with epilepsy were more likely to have positive/likely positive findings compared with the nonepilepsy group (38.2 vs. $28.7 \%$; $P=0.004$; Table 3 ). The epilepsy group had a higher diagnostic yield in characterized disease genes compared with the nonepilepsy group (33.4 vs. $25.9 \%$; $P=0.015$; Table 3 ). Overall detection of novel genetic etiologies did not differ significantly between the two groups; however, the epilepsy group was more likely to have likely positive findings in novel genetic
Table 3 Overall positive, uncertain, novel, and negative detection rates

\begin{tabular}{|c|c|c|c|c|c|}
\hline \multirow[b]{2}{*}{ Overall positive ${ }^{a}$} & \multicolumn{2}{|c|}{$\begin{array}{l}\text { Epilepsy } \\
\text { group }\end{array}$} & \multicolumn{2}{|c|}{$\begin{array}{l}\text { Nonepilepsy } \\
\text { group }\end{array}$} & \multirow{2}{*}{$\begin{array}{c}P \\
\begin{array}{c}P \\
\text { value }\end{array} \\
0.004\end{array}$} \\
\hline & $112 / 293$ & $38.2 \%$ & $210 / 732$ & $28.7 \%$ & \\
\hline Characterized genes & $105 / 314$ & $33.4 \%$ & $212 / 817$ & $25.9 \%$ & 0.015 \\
\hline Positive & 79/314 & $25.2 \%$ & $167 / 817$ & $20.4 \%$ & 0.091 \\
\hline Likely positive & $26 / 314$ & $8.3 \%$ & $45 / 817$ & $5.5 \%$ & 0.100 \\
\hline $\begin{array}{l}\text { Novel genetic } \\
\text { etiologies }^{b}\end{array}$ & $18 / 200$ & $9.0 \%$ & $36 / 521$ & $6.9 \%$ & 0.345 \\
\hline Likely positive & $14 / 200$ & $7.0 \%$ & $17 / 521$ & $3.3 \%$ & 0.038 \\
\hline Possibly positive & $4 / 200$ & $2.0 \%$ & 19/521 & $3.6 \%$ & 0.346 \\
\hline $\begin{array}{l}\text { Uncertain } \\
\text { (characterized genes) }\end{array}$ & $34 / 314$ & $10.8 \%$ & $80 / 817$ & $9.8 \%$ & 0.58 \\
\hline Negative $^{a}$ & $144 / 293$ & $49.1 \%$ & $431 / 732$ & $58.9 \%$ & 0.005 \\
\hline
\end{tabular}

averall positive includes positive/likely positive findings in characterized genes and likely positive novel genetic etiologies; it does not include 21 probands from the epilepsy group and 85 probands from the nonepilepsy group in whom only characterized disease genes were analyzed ("first-tier" analysis). "Includes only probands in whom analysis of both characterized and novel genetic etiologies was performed.

etiologies than the nonepilepsy group ( 7.0 vs. $3.3 \%$; $P=0.038$; Table 3).

\section{Logistic regression to account for differences between cohorts}

The epilepsy group had significantly higher rates of intellectual disability/developmental delay, autism, abnormal brain magnetic resonance imaging findings, and family history of seizures. We therefore investigated whether these factors may be responsible for the higher diagnostic rate in patients with epilepsy. Logistic regression analysis adjusting for potential confounding effects did not affect the overall results and revealed an odds ratio of 1.50 (95\% confidence interval $=1.11-2.02 ; P=0.008)$ for positive DES results. We then used the same model to assess the probability of receiving a negative result from exome sequencing, which we considered a more relevant measure in clinical practice. Logistic regression analysis adjusting for potential confounding variables revealed that patients in the epilepsy group were less likely to receive negative results from DES than patients in the nonepilepsy group (odds ratio $=0.67$; $95 \%$ confidence interval $=0.50-0.89 ; P=0.006)$.

\section{Positive rates by epilepsy diagnosis and age of seizure onset}

When the overall DES positive rate was examined based on epilepsy diagnosis, patients with epileptic encephalopathies and generalized epilepsies had the highest rates of positive/ likely positive findings from DES (Supplementary Table S4 and Supplementary Figure S2 online). Furthermore, patients with epileptic encephalopathies and generalized epilepsies accounted for nearly all novel genetic etiology discoveries. Likely positive novel genetic etiologies were identified in $17 \%(11 / 65)$ of all patients with epileptic encephalopathies in whom novel genetic etiologies were analyzed and accounted 
for $25 \%(9 / 36)$ of all positive findings in this patient subset. Among patients with epileptic encephalopathies, the highest positive rate was observed in patients with neonatal-onset epileptic encephalopathies (58.3\%; Supplementary Table S5 online). Infantile spasms, which comprised the largest patient subset among the epileptic encephalopathies, had an overall DES positive rate of $39.0 \%$ (Supplementary Table S5). Novel genetic etiologies were identified in $6 / 36$ (16.7\%) patients with infantile spasms and accounted for $37.5 \%$ of the positive findings in this patient subset; all the novel genetic etiologies identified in patients with infantile spasms were classified as likely positive. Overall DES detection rates calculated by age of seizure onset are summarized in Supplementary Table S5. Patients with neonatal-onset seizures were most likely to have positive findings on DES, with an overall positive rate of $42.9 \%$.

\section{Inheritance patterns among positive findings}

Among the 119/314 positive/likely positive epilepsy cases, 122 pathogenic/likely pathogenic alterations were identified; among the $229 / 817$ positive/likely positive nonepilepsy cases, 240 pathogenic/likely pathogenic alterations were identified. Inheritance patterns for pathogenic/likely pathogenic molecular findings for the epilepsy group are summarized in Table 4 (data for the nonepilepsy group are summarized in Supplementary Table $\mathbf{S 6}$ online). The majority of positive DES findings among all patients were the result of de novo mutations. Patients with epilepsy were more likely to have pathogenic/likely pathogenic de novo mutations than patients in the nonepilepsy group (67.9 vs. $53.3 \%$; $P=0.013$; Supplementary Table S6).

Table 4 Inheritance patterns among 122 positive/likely positive gene findings from 119 patients with seizures

\begin{tabular}{lcc} 
& Epilepsy group \\
\hline Autosomal dominant & $\mathbf{7 9}$ & $\mathbf{6 4 . 8} \%$ \\
De novo & 65 & $53.3 \%$ \\
Inherited from affected parent & 6 & $4.9 \%$ \\
Pathogenic mutation (reduced penetrance) & 1 & $0.8 \%$ \\
Inherited from unaffected mosaic parent & 1 & $0.8 \%$ \\
Inherited from unaffected parent with imprinting & 0 & $0.0 \%$ \\
Unknown & 6 & $4.9 \%$ \\
Autosomal recessive & 24 & $19.7 \%$ \\
Inherited & 23 & $18.9 \%$ \\
Unknown, likely inherited & 1 & $0.8 \%$ \\
X-linked recessive & 10 & $8.2 \%$ \\
De novo & 4 & $3.3 \%$ \\
Maternally inherited & 6 & $4.9 \%$ \\
Unknown & 0 & $0.0 \%$ \\
X-linked dominant & 9 & $7.4 \%$ \\
De novo & 7 & $5.7 \%$ \\
Inherited from affected mother & 0 & $0.0 \%$ \\
Unknown, likely de novo & 2 & $1.6 \%$ \\
\hline
\end{tabular}

\section{Results in epileptic encephalopathies}

When analyzing the inheritance patterns of the pathogenic/ likely pathogenic mutations stratified by epilepsy diagnosis, we found that the highest rate of causative de novo mutations was observed in patients with epileptic encephalopathies (Supplementary Table S6). In this patient subset, nearly $75 \%$ (30/41) of pathogenic or likely pathogenic mutations arose de novo in either autosomal or $\mathrm{X}$ chromosome genes. When considering the entire subset of 89 patients with epileptic encephalopathies, pathogenic de novo mutations were identified in $33.7 \%(30 / 89)$ patients. Furthermore, 17\% (7/41) of the pathogenic mutations identified in patients with epileptic encephalopathies were consistent with autosomal recessive inheritance, which accounts for $7.9 \%$ (7/89) of the entire subset of patients with epileptic encephalopathies. Maternally inherited X chromosome mutations were identified in three male probands with epileptic encephalopathies, accounting for $3 / 41$ (7.3\%) pathogenic mutations and 3/89 (3.4\%) total cases with epileptic encephalopathies.

\section{Characterization of molecular findings}

Of the 119 positive/likely positive cases, 105 (88.2\%) patients were found to have pathogenic/likely pathogenic mutations in previously characterized disease genes (Table 5; Supplementary Table S3 online). Mutations were identified in a total of 93 unique genes, 21 of which were found in two or more unrelated patients (Table 5). Mutations in six genes (KCNQ2, MECP2, FOXG1, IQSEC2, KMT2A, and STXBP1) were the most frequent findings, with mutations in each of these genes identified in three or more unrelated probands. Of the 119 patients with positive/likely positive findings, 3 had pathogenic/likely pathogenic alterations identified in two genes (Supplementary Table S3 online). In one of these patients, two significant findings were associated with nonoverlapping phenotypic presentations (patient 73). The significant genetic findings in the other two patients (patients 6 and 14) possibly contributed to the major clinical indications for testing.

Of the 119 positive/likely positive cases, 14 (11.8\%) had novel genetic etiology discoveries (Table 5; Supplementary Table S7 and Supplementary Materials and Methods online). Furthermore, four additional probands had possibly positive novel genetic etiology findings (Supplementary Table S7). Altogether, novel genetic etiologies were identified in 18 probands, representing 21 novel disease genes, with three probands each having two identified novel genetic etiologies. Of the 21 novel genes identified, $3 / 21(14.3 \%)$ were subsequently confirmed as recognized disease genes in independent publications-COQ4 (refs. 33,34), DNM1 (ref. 16), and PURA ${ }^{35,36}$ after the clinical DES report was issued. The authors of these publications were unaware of our reports, and our patients were not included in the respective publications. The median time from clinical report to independent publication was 16 weeks (Supplementary Table S7). In addition to these confirmed disease genes, 6/21 (28.6\%) clinically novel genes, including 
Table 5 Positive/likely positive genes identified in 119 patients with seizures

\begin{tabular}{|c|c|c|}
\hline Characterized disease genes & Occurrences & Frequency \\
\hline KCNQ2, MECP2 & 4 & $1.27 \%$ \\
\hline FOXG1, IQSEC2, KMT2A, STXBP1 & 3 & $0.96 \%$ \\
\hline $\begin{array}{l}\text { ANKRD11, AP4B1, ASXL1, ATP1A3, CACNA1A, CHD2, CUL4B, EEF1A2, GNAO1, GRIN1, MEF2C, SCN1A, SCN2A, TPP1, } \\
\text { WDR45 }\end{array}$ & 2 & $0.64 \%$ \\
\hline $\begin{array}{l}\text { ACO2, ACTB, ALX4, ANO3, ARHGEF9, ATP2B3, BRAF, CACNA1C, CASK, CCND2, CHD7, COL4A1, CREBBP, CTNNB1, } \\
\text { DARS2, DEAF1, DYNC1H1, DYRK1A, FGFR2, FHL1, GLI2, GRHL3, GRIN2B, HMBS, KAT6B, KCNC3, KCTD7, LDLR, LINS, } \\
\text { MAP2K1, NALCN, NDUFA1, NDUFAF2, NDUFB3, NGLY1, NOTCH1, PACS1, PANK2, PKD1, PLP1, POMC, PPT1, PRNP, RARS2, } \\
\text { RNF213, SCN3A, SMAD2, SMC1A, SPAST, SYNGAP1, TBCE, TBX19, TCTN2, TUBA1A, TUBB4A, UBE3A, WAC, ZNF335 }\end{array}$ & 1 & $0.32 \%$ \\
\hline Novel genetic etiologies & Occurrences & Frequency \\
\hline \multicolumn{3}{|l|}{ Likely positive $^{a}$} \\
\hline $\begin{array}{l}\text { AIMP2, CACNA1E, CDC42, CLTC, COQ4 }{ }^{\mathrm{b}}, \text { DGKZ, DNM1 b , HDAC1, HDAC3, HNRNPR, HTR2C, PURA }{ }^{\mathrm{b}}, \text { SNAP25, SON } \\
\text { Possibly positive }\end{array}$ & 1 & $0.32 \%$ \\
\hline ABCA5, LPHN1, LRFN2, MN1, OGT, SV2A, TIMM50 & 1 & $0.32 \%$ \\
\hline
\end{tabular}

apatient with AIMP2 alteration also has alteration in $L R F N 2$; patient with $D G K Z$ alteration also has alteration in $A B C A 5$; patient with HDAC3 alteration also has alteration in LPHN1. ${ }^{\circ}$ The clinical significance of these genes was corroborated by subsequent independent publications with additional patients with similar clinical presentations.

CACNA1E, CLTC, OGT, SNAP25, SON, and SV2A, were subsequently reported in large-scale research studies in single patients (Supplementary Table S7). ${ }^{11,37,38}$

\section{DISCUSSION}

\section{Summary}

In our study, we examined the rate of positive findings of DES in patients with epilepsy when compared with other presumed genetic conditions without epilepsy. Positive findings were identified in $38 \%$ of patients with epilepsy versus $29 \%$ of patients without epilepsy. This nearly $10 \%$ excess in positive findings suggests that DES may be an effective tool in identifying the causative molecular defects in this patient population. Patients with epilepsy were 1.5 times more likely to receive positive DES results than patients without epilepsy.

\section{Diagnostic yield}

We observed that DES was more likely to identify a pathogenic mutation in patients with neonatal-onset epileptic encephalopathies $(58.3 \%)$ compared with patients with later onset. In addition, in patients with epileptic encephalopathies with onset in the first year of life, the overall positive rate was $41.8 \%$, which is in line with recent smaller studies examining the diagnostic yield of targeted NGS panels. ${ }^{39}$ This finding suggests that the increased yield in patients with epilepsy is driven largely by patients with neonatal and infantile onset. Even though several genes, such as KCNQ2, are already established, this particular group has not been systematically investigated in existing studies. Accordingly, our study may help shed some light on the genetic architecture of this patient population. Twenty-one genes were identified in multiple unrelated probands in our cohort. Many of these genes (e.g., KCNQ2, MECP2, SCN1A, $S C N 2 A$, and $S T X B P 1$ ) are well established for severe early-onset epilepsies. Other recurrent genes, such as EEF1A2, GNAO1, and GRIN1, have only recently been described in patients with severe epilepsies, and their phenotypic spectrum and exact role remain to be fully elucidated. The identification of mutations in these genes in multiple patients from an unselected laboratory cohort suggests that they may play an important role in epilepsy. In addition, pathogenic mutations in epilepsy-associated genes, including FOXG1, GNAO1, GRIN2B, SCN8A, and STXBP1, were identified in patients in our cohort with neurodevelopmental disorders without seizures, lending further evidence to the broad phenotypic spectrum and shared pathways of intellectual disability, autism, movement disorders, and epilepsy.

\section{Discovery of novel genetic etiologies in the diagnostic setting}

Our study systematically assessed novel genes as well as novel diseases in characterized disease genes for their potential role in disease etiology, a strategy that we refer to as "diagnostic novel gene reporting." We identified 14 likely positive novel genetic etiologies in 14 separate patients with epilepsy, representing $7 \%$ of all patients with epilepsy who underwent this type of analysis. Although these genes have not yet been definitively implicated in disease because of their novelty, we were motivated to address these findings given the rapidly evolving field with frequent gene discoveries. Of the 65 patients in our cohort with epileptic encephalopathies who underwent analysis of novel genetic etiologies, 9 (14\%) were found to have likely positive novel genetic etiologies. Six of these patients had infantile spasms, and three had other neonatal or infantile-onset epileptic encephalopathies. Our data suggest that novel genetic etiologies may play a particularly important role in the epileptic encephalopathies, especially in patients with seizure onset in the first year of life. Likely causative novel genetic etiologies were identified in significantly more patients with epilepsy than patients without epilepsy, further supporting the utility of DES in this patient population.

\section{Validation of novel genetic etiologies}

To ascertain whether our strategy for the reporting of diagnostic novel genetic etiologies has validity, we examined the probability that novel genetic etiologies were subsequently supported 
by reports in independent studies of additional patients with similar clinical presentations and alterations in the same genes. Three genes in our epilepsy cohort (COQ4, DNM1, and PURA), accounting for $14 \%(3 / 21)$ of all novel genetic etiologies identified in patients with epilepsy, were subsequently confirmed in independent publications. ${ }^{16,33-36}$ Although it is difficult to assess to what extent this frequency is higher than would be expected by chance, we feel reassured by the fact that approximately $15 \%$ of all genes that were reported as potential novel disease genes received subsequent independent confirmation without knowledge of our prior report. This number may provide a baseline for future studies examining the role of diagnostic novel gene reporting. In addition to genes that were independently validated, several novel genes reported as likely causative were also found in individual patients in large cohort studies of patients with neurodevelopmental disorders. ${ }^{11,37,38}$ This observation was made for $29 \%$ of the novel genetic etiologies identified in our entire cohort, including six genes (CACNA1E, CLTC, OGT, SNAP25, SON, and $S V 2 A$ ). The relevance of this finding is currently not known, but we assume that some of these genes may also become definitively implicated causes of genetic epilepsies. Taking these findings together, systematically analyzing novel genes found only in single individuals has limitations. However, this approach may be necessitated by the highly heterogeneous genetic architecture of childhood epilepsies and the increasing use of exome sequencing in a diagnostic context. Further development of guidelines for this particular type of analysis will help advance the field to address the role of alterations in genes only found in single individuals.

\section{Role of various inheritance models}

Our results also provide an interesting glimpse of the genetic architecture of epilepsies, particularly the epileptic encephalopathies, which comprise the largest subset of patients in our cohort. In line with recent studies, ${ }^{15,16,38}$ the majority of positive cases with epileptic encephalopathies in our cohort were the result of de novo mutations, either in autosomal or $\mathrm{X}$ chromosome genes. De novo mutations explain nearly $75 \%$ of positive findings in this patient cohort and account for one-third of patients with epileptic encephalopathies overall. However, nearly $20 \%$ of the positive findings among patients with epileptic encephalopathies in our cohort were due to autosomal recessive inheritance. Another 7\% of positive cases were the result of $\mathrm{X}$-linked recessive inheritance. Altogether, these findings suggest that autosomal recessive and $\mathrm{X}$-linked recessive inheritance may explain approximately $10 \%$ of all cases of epileptic encephalopathy. Even though our patient cohort may have been biased toward cases with recessive disease, our findings raise the issue that, in addition to the undisputed importance of de novo mutations, inherited mutations may play a significant role in the epileptic encephalopathies.

\section{Limitations}

There are various limitations to our current study. First, it is not clear to what extent our patient population is representative of epilepsy. In some cases, patients underwent exome sequencing only after a long diagnostic journey. However, in other patients, exome sequencing was performed as the first molecular diagnostic test. A significant portion of patients included in our study had prior genetic testing, including an uninformative genome-wide array in $80 \%$ of patients and negative gene panel testing in $30 \%$ of patients. Although it is difficult to assess to what degree prior negative testing may have influenced our results, it is important to emphasize that our referral-based patient cohort is biased and not population-based. However, we feel that this heterogeneity is representative of the clinical scenarios that a neurologist, medical geneticist, or genetic counselor is confronted with in clinical practice. Therefore, although our results may be applicable to a typical clinical situation in which patients with various levels of previous testing are encountered, they may not fully reflect the genetic architecture of all human epilepsies.

Another limitation of our study was the lack of phenotypic detail for many patients, which is a common issue for many laboratory-based studies. We tried to classify the epilepsy phenotypes into established categories but were unable to assign a definite epilepsy syndrome in a large proportion of patients. However, for most patients the data were sufficient to assess age of onset, findings on neuroimaging, and comorbidities. Given that the phenotype in many recently discovered genetic epilepsies may be complex, and that the spectrum still needs to be defined, we did not pursue detailed characterization, and, in many cases, we connected clinicians with researchers in the field who pursue characterization of the specific phenotype.

Taken together, our data from 314 unselected patients with epilepsy indicate that DES, with analysis and interpretation of novel genetic etiologies, is particularly useful in patients with severe early-onset epilepsies, exceeding the yield for many other neurodevelopmental disorders and epilepsies with a later age of onset. We hope these data are useful for establishing professional guidelines for genetic testing for patients with epilepsy.

\section{SUPPLEMENTARY MATERIAL}

Supplementary material is linked to the online version of the paper at http://www.nature.com/gim

\section{ACKNOWLEDGMENTS}

We are grateful to the patients and their families for their participation and to their physicians and genetic counselors for providing samples and clinical histories.

\section{DISCLOSURE}

K.L.H., K.D.F., D.N.S., C.M., Z.P., S.L., and S.T. are employed by and receive a salary from Ambry Genetics. Exome sequencing is among its commercially available tests. I.H. declares no conflict of interest.

\section{REFERENCES}

1. Biesecker LG, Green RC. Diagnostic clinical genome and exome sequencing. N Engl J Med 2014;370:2418-2425

2. Shashi V, McConkie-Rosell A, Rosell B, et al. The utility of the traditional medical genetics diagnostic evaluation in the context of next-generation sequencing for undiagnosed genetic disorders. Genet Med 2014;16:176-182. 
3. Soden S, Saunders CJ, Willig, LK, et al. Effectiveness of exome and genome sequencing guided by acuity of illness for diagnosis of neurodevelopmental disorders. Sci Trans/ Med 2014;6:265ra168.

4. ACMG Board of Directors. Points to consider in the clinical application of genomic sequencing. Genet Med 2012;14:759-761.

5. Yang Y, Muzny DM, Reid JG, et al. Clinical whole-exome sequencing for the diagnosis of mendelian disorders. N Engl J Med 2013;369:1502-1511.

6. Yang Y, Muzny DM, Xia F, et al. Molecular findings among patients referred for clinical whole-exome sequencing. JAMA 2014;312:1870-1879.

7. Farwell KD, Shahmirzadi L, El-Khechen D, et al. Enhanced utility of familycentered diagnostic exome sequencing with inheritance model-based analysis: results from 500 unselected families with undiagnosed genetic conditions. Genet Med 2015;17:578-586.

8. Dixon-Salazar TJ, Silhavy JL, Udpa N, et al. Exome sequencing can improve diagnosis and alter patient management. Sci Trans/ Med 2012;4:138ra178.

9. Srivastava $\mathrm{S}, \mathrm{C}$ Chen JS, Vernon $\mathrm{H}$, et al. Clinical whole exome sequencing in child neurology practice. Ann Neurol 2014;76:473-483.

10. Rauch A, Wieczorek D, Graf E, et al. Range of genetic mutations associated with severe non-syndromic sporadic intellectual disability: an exome sequencing study. Lancet 2012;380:1674-1682.

11. de Ligt J, Willemsen $\mathrm{MH}$, van Bon BW, et al. Diagnostic exome sequencing in persons with severe intellectual disability. N Engl J Med 2012;367:1921-1929.

12. Geschwind DH, State MW. Gene hunting in autism spectrum disorder: on the path to precision medicine. Lancet Neuro/ 2015;14:1109-1120.

13. Helbig I, Lowenstein DH. Genetics of the epilepsies: where are we and where are we going? Curr Opin Neuro/ 2013;26:179-185

14. Thomas RH, Berkovic SF. The hidden genetics of epilepsy-a clinically important new paradigm. Nat Rev Neuro/ 2014;10:283-292.

15. Epi4K Consortium, Epilepsy Phenome/Genome Project, Allen AS, et al. De novo mutations in epileptic encephalopathies. Nature 2013;501:217-221.

16. EuroEPINOMICS-RES Consortium, Epilepsy Phenome/Genome Project, Epi4K Consortium. De novo mutations in synaptic transmission genes including DNM1 cause epileptic encephalopathies. Am J Hum Genet 2014;95:360-370.

17. O'Roak BJ, Deriziotis $P$, Lee $C$, et al. Exome sequencing in sporadic autism spectrum disorders identifies severe de novo mutations. Nat Genet 2011;43:585-589.

18. O'Roak BJ, Stessman HA, Boyle EA, et al. Recurrent de novo mutations implicate novel genes underlying simplex autism risk. Nat Commun 2014;5:5595.

19. Chénier S, Yoon G, Argiropoulos B, et al. CHD2 haploinsufficiency is associated with developmental delay, intellectual disability, epilepsy and neurobehavioural problems. J Neurodev Disord 2014;6:9

20. Lemke JR, Hendrickx R, Geider K, et al. GRIN2B mutations in West syndrome and intellectual disability with focal epilepsy. Ann Neurol 2014;75:147-154.

21. Carvill GL, Heavin SB, Yendle SC, et al. Targeted resequencing in epileptic encephalopathies identifies de novo mutations in CHD2 and SYNGAP1. Nat Genet 2013;45:825-830.

22. Nakamura K, Kato M, Osaka H, et al. Clinical spectrum of SCN2A mutations expanding to Ohtahara syndrome. Neurology 2013;81:992-998.

23. Iglesias A, Anyane-Yeboa K, Wynn J, et al. The usefulness of whole-exome sequencing in routine clinical practice. Genet Med 2014;16:922-931.

24. Veeramah KR, Johnstone L, Karafet TM, et al. Exome sequencing reveals new causal mutations in children with epileptic encephalopathies. Epilepsia 2013;54:1270-1281.

25. Martin HC, Kim GE, Pagnamenta AT, et al.; WGS500 Consortium. Clinical whole-genome sequencing in severe early-onset epilepsy reveals new genes and improves molecular diagnosis. Hum Mol Genet 2014;23:3200-3211.

26. Dimassi S, Labalme A, Ville $D$, et al. Whole-exome sequencing improves the diagnosis yield in sporadic infantile spasm syndrome. Clin Genet; e-pub ahead of print 3 July 2015
27. Berg AT, Berkovic SF, Brodie MJ, et al. Revised terminology and concepts for organization of seizures and epilepsies: report of the ILAE Commission on Classification and Terminology, 2005-2009. Epilepsia 2010;51:676-685.

28. Gnirke A, Melnikov A, Maguire J, et al. Solution hybrid selection with ultra-long oligonucleotides for massively parallel targeted sequencing. Nat Biotechnol 2009;27:182-189.

29. Ye K, Schulz MH, Long Q, Apweiler R, Ning Z. Pindel: a pattern growth approach to detect break points of large deletions and medium sized insertions from paired-end short reads. Bioinformatics 2009;25:2865-2871.

30. LaDuca H, Stuenkel AJ, Dolinsky JS, et al. Utilization of multigene panels in hereditary cancer predisposition testing: analysis of more than 2,000 patients. Genet Med 2014;16:830-837.

31. Richards S, Aziz N, Bale S, et al.; ACMG Laboratory Quality Assurance Committee. Standards and guidelines for the interpretation of sequence variants: a joint consensus recommendation of the American College of Medical Genetics and Genomics and the Association for Molecular Pathology. Genet Med 2015;17:405-424.

32. R Development Core Team. (2008) R: A Language and Environment for Statistical Computing. R Foundation for Statistical Computing. http://www.Rproject.org. Accessed on 18 November 2014.

33. Brea-Calvo G, Haack TB, Karall D, et al. COQ4 mutations cause a broad spectrum of mitochondrial disorders associated with CoQ10 deficiency. Am J Hum Genet 2015;96:309-317.

34. Chung WK, Martin K, Jalas C, et al. Mutations in COQ4, an essential component of coenzyme $\mathrm{Q}$ biosynthesis, cause lethal neonatal mitochondrial encephalomyopathy. J Med Genet 2015;52:627-635.

35. Hunt D, Leventer RJ, Simons $C$, et al.; DDD study. Whole exome sequencing in family trios reveals de novo mutations in PURA as a cause of severe neurodevelopmental delay and learning disability. J Med Genet 2014;51: 806-813.

36. Lalani SR, Zhang J, Schaaf CP, et al. Mutations in PURA cause profound neonatal hypotonia, seizures, and encephalopathy in 5 q31.3 microdeletion syndrome. Am J Hum Genet 2014;95:579-583.

37. O'Roak BJ, Vives L, Girirajan S, et al. Sporadic autism exomes reveal a highly interconnected protein network of de novo mutations. Nature 2012;485: 246-250.

38. Deciphering Developmental Disorders Study. Large-scale discovery of novel genetic causes of developmental disorders. Nature 2015;519:223-228.

39. Mercimek-Mahmutoglu S, Patel J, Cordeiro D, et al. Diagnostic yield of genetic testing in epileptic encephalopathy in childhood. Epilepsia 2015;56: 707-716. 